\title{
Les blogs de science dans la recherche et la médiation scientifiques : pourquoi, comment et pour qui ?
}

par Antoine Blanchard

La révolution du web 2.0, qui vit internet passer d'une dynamique de diffusion à une dynamique d'interaction où chaque internaute peut exister et être reconnu par les conversations auxquelles il participe sur diverses plateformes et réseaux sociaux numériques, n'a pas épargné la communauté scientifique. En particulier, le format «blog » (terme formé par aphérèse de l'expression « web $\log »$, pour journal en ligne), initialement utilisé pour des journaux intimes, s'est développé comme un outil de publication et de conversation répondant aux besoins variés de cette communauté.

D'abord isolés, puis regroupés en communautés [Blanchard, 2016], les blogueurs de science (expression qui inclut les scientifiques professionnels mais aussi les étudiants, journalistes scientifiques, amateurs de science, musées et centres de science, groupes concernés... qui tiennent un blog à teneur scientifique) ont démontré leur capacité à influencer la manière dont la recherche avance, dont les résultats sont communiqués et dont les publics sont touchés. Parmi les nombreux sujets dont les blogs discutent, je me concentrerai ici sur les sujets proprement scientifiques ou liés au monde de la recherche, délimitant ainsi une sous-catégorie du «blogging scientifique » qui fait du numérique académique ( « digital scholarship ») une conversation ( « conversational scholarship ») [Gregg, 2006]. En quoi cela consiste-t-il concrètement ? Où cette évolution nous entraine-t-elle ? Je tenterai de faire la lumière sur ce phénomène dans ce chapitre.

\section{Pourquoi bloguer?}

En 2008, un questionnaire informel intitulé «Why blog ? », issu de la communauté anglophone Scienceblogs ${ }^{1}$, a circulé pour interroger les blogueurs sur leurs motivations. Plusieurs douzaines de blogs de science anglophones et francophones ont ainsi répondu au questionnaire sur leur blog. Leurs réponses apportent une vision relativement large des raisons couramment admises de bloguer sur la science, dont trois ressortent en particulier.

En premier lieu, les blogueurs de science reçoivent une gratification immédiate dans le sens où leur blog devient leur identité en ligne, laquelle reflète leur expertise, leur passion, et leur volonté de s'engager dans le débat. Elle leur apporte une reconnaissance, qui peut être réticulaire lorsqu'ils laissent des commentaires sur d'autres blogs. Elle améliore également leur référencement par les moteurs de recherche et ouvre la possibilité d'atteindre un certain niveau de notoriété. Cette visibilité sur internet se transpose au «monde physique » au fur et à mesure que le blog croit en crédibilité : nombreux sont les exemples de blogueurs de science qui ont été invités par des médias traditionnels à commenter une actualité, ou par des collègues à intervenir devant un parterre d'experts ou de profanes. La présence en ligne s'incarnait autrefois dans des sites web personnels qui incluaient dans le meilleur des cas un formulaire de contact, remplacé aujourd'hui par des outils de blog plus interactifs et plus faciles à prendre en main. Une fois présent sur le web, un blogueur peut commencer à construire son réseau de communication, en combinant l'utilisation du blog avec une panoplie de réseaux sociaux numériques orientés vers la production, la diffusion et/ou l'organisation de l'information selon ses goûts personnels. (Pour prendre un exemple fictif permettant d'illustrer la diversité de ces usages : un chercheur peut faire un usage professionnel du

\footnotetext{
${ }^{1}$ http://scienceblogs.com
} 
« microblogging $»^{2}$ pour tenir ses collègues informés de ses activités et organiser des rencontres lors de congrès internationaux ; utiliser un outil social de gestion bibliographique ${ }^{3}$ pour organiser et partager des articles scientifiques ; commenter des articles publiés pour témoigner notamment des difficultés à en reproduire les résultats ${ }^{4}$; tenir son cahier de laboratoire sur un wiki collaboratif ${ }^{5} \ldots$...). Les blogueurs voient dans ces outils la version numérique du réseautage informel à la machine au café ou du « journal club » du laboratoire, à une échelle plus large et avec une plus grande diversité d'activités. Autrement dit, ils sont généralement bien conscients que les blogs de science sont un outil additionnel pour les esprits curieux, et non un remplacement miraculeux du travail long et fastidieux, demandant volonté et patience, inhérent à la pratique scientifique.

Ensuite, les blogueurs de science s'efforcent de combler ce qu'ils perçoivent comme un manque d'ouverture et de transparence de la recherche. Classiquement, la science est représentée comme une progression linéaire vers la connaissance à travers la discussion logique et constante. À l'inverse, les blogueurs de science tentent de montrer d'autres facettes du travail de laboratoire et de rendre compte du processus dynamique de la recherche en action, vue depuis la ligne de front plutôt qu'à travers sa reconstitution publiée ou avec le recul historique de la « science déjà faite ». En créant un forum ouvert de pairs, ils insèrent les blogs de science dans le mouvement plus large de la science ouverte, qui inclut des initiatives comme l'accès libre (revues en libre accès ou archives ouvertes), cahiers de laboratoire ouverts, évaluation ouverte par les pairs... Même si les nombreux enjeux de la science ouverte débordent du cadre de cet article, il est intéressant de noter que les blogs de science résolvent l'un des problèmes soulevés par le célèbre physicien Richard Feynman dans sa conférence Nobel en 1965 [Feynman, 1965] : « Nous avons l'habitude d'écrire des articles publiés dans des revues scientifiques pour rendre le travail le plus abouti possible, pour couvrir nos traces, pour ne pas nous soucier des points aveugles ou de comment la première idée que nous avons eue s'est avérée fausse, etc. Ainsi il n'existe pas d'endroit où publier, de manière digne, ce que nous avons réellement fait dans notre travail. » Sur les blogs de science, ces informations peuvent trouver leur juste place.

Troisièmement, s'adresser au public le plus large possible est une priorité des blogueurs de science qui, en écho au diagnostic de Michel Claessens à la DG Recherche de la Commission européenne [Claessens, 2009], sont souvent insatisfaits de la manière ou de la fréquence à laquelle la science est traitée dans les médias généralistes. De nombreux blogueurs de science se perçoivent comme des éducateurs, essayant de relever le niveau des discussions sur des thèmes comme le changement climatique ou l'évolution [Goldstein, 2009]. En France par exemple, le journaliste scientifique Sylvestre Huet qui publiait de courts articles par numéro du journal Libération, a utilisé son blog pour traiter plus longuement de la controverse sur les causes du changement climatique et sur le rôle du soleil. Ce battage médiatique, amplifié par d'autres blogs, fut déclenché par un débat à l'Académie des sciences. Les chercheurs qui s'engagent auprès du grand public répondent à une demande claire : selon le sondage Eurobaromètre de décembre 2007, « la majorité de la population de l'Europe des 27 préfère que les scientifiques (à $52 \%$ ), plutôt que les journalistes (à 14 \%), leur présentent l'actualité scientifique » [Commission européenne, 2007].

Les autres motivations des blogueurs de science, beaucoup moins explicites dans les réponses au questionnaire, recouvrent d'autres considérations importantes liées à la liberté éditoriale et la gestion de ses connaissances personnelles. En effet, les blogueurs estiment que les espaces numériques qu'ils créent leur permettent de regagner un peu du pouvoir que la communauté a cédé aux éditeurs scientifiques et aux organismes de recherche. Là ils décident de leur politique éditoriale, définissent leurs propres règles et au-delà de leurs écrits, ils fabriquent un outil de

\footnotetext{
${ }^{2}$ Par exemple http://twitter.com/

${ }^{3}$ Par exemple http://www.citeulike.org

${ }^{4}$ Par exemple http://pubpeer.com

${ }^{5}$ Par exemple http://openwetware.org
} 
recommandation qui renvoie vers des articles scientifiques, des blogs (qu'on retrouve classiquement dans la «blogoliste » affichée dans un colonne latérale) ou tout autre référence en ligne ou hors ligne. Peu importe l'impératif de préservation des sources du savoir scientifique, un blogueur peut très bien décider de publier des contenus plus éphémères dans le flux du web et inventer de nouvelles formes d'expression, à l'instar des outils de «microblogging » comme Twitter ${ }^{6}$. Plusieurs des blogueurs ayant répondu au questionnaire sur leurs motivations ont indiqué que leur blog leur servait à clarifier leurs idées et qu'en poussant leurs investigations sur un nouveau sujet au point de pouvoir en tirer un billet de blog, ils apprenaient et mémorisaient mieux. En outre, une fois qu'ils avaient blogué sur un sujet, il leur était plus facile de retrouver et réutiliser l'information qu'ils avaient acquise, que s'ils avaient simplement ajouté une page internet en favori ou en avaient enregistré une copie.

Après cette introduction générale aux blogs de science, je souhaite maintenant pénétrer les deux genres qui les constituent : bloguer pour la recherche et bloguer pour la médiation.

\section{Bloguer pour la recherche}

L'expression «bloguer pour la recherche » renvoie au fait de bloguer son travail pour soi-même et pour les autres scientifiques. Ce processus est souvent confondu avec le fait de « documenter sur un blog sa recherche », c'est-à-dire de rendre compte de l'avancée de ses réflexions ou expériences, en mêlant des considérations méthodologiques, des raisonnements logiques et des résultats bruts. Or, comme les détails expérimentaux et les faits les plus ardus sont souvent omis, on serait conduits à tort à considérer cette documentation comme imparfaite. Essayons plutôt de ne pas confondre la carte et le territoire : comme l'a expliqué la blogueuse Jill Walker en 2003 déjà ${ }^{7}$, « les blogs ne servent pas à documenter mais à faire, penser et discuter. Ils attrapent des idées en vol pour les rendre explicites » [Walker, 2006]. Des chercheurs bloguent leurs nouvelles idées ou leurs réponses à des travaux publiés quand ils considèrent qu'une publication traditionnelle n'est pas nécessaire, ou prendrait trop de temps (exemple des courriers au comité éditorial). De nombreuses revues à large lectorat et de l'ère pré-internet comme Nature, Science, Cell... ont, depuis 2006, lancé des blogs officiels pour multiplier leur présence en ligne ${ }^{8}$. En ce qui concerne les revues lancées à l'ère du web 2.0, elles offrent des services de blogs hébergés à la communauté ${ }^{9}$, afin de fidéliser leurs auteurs et lecteurs, et de capter cette manne qui complète les contenus « certifiés » des revues à comité de lecture. Les blogs de science, propices aux échanges interdisciplinaires et à la diffusion large et rapide d'idées scientifiques, sont au service de la science en train de se faire.

Bien sûr, tous les nouveaux réseaux de communication entre professionnels de la recherche ne sont pas des blogs, mais ils mobilisent des principes similaires. Prenons les listes de diffusion : un exemple datant de 2009 montre que des échanges initiés au sein d'une communauté spécialisée peut agréger des personnes aussi diverses que des étudiants, post-doctorants, et directeurs d'équipes de recherche du monde entier ${ }^{10}$, et ce en l'espace d'une semaine seulement. Cet échange vif concernant un sujet technique lié à une biobanque de mutants bactériens a même impliqué les prix Nobel Paul Nurse et Tim Hunt !

Le premier objectif du blog de science, comme je l'ai mentionné, est souvent de se rendre visible et d'être lu, ou du moins remarqué. Dans un monde académique où les personnes se jugent en fonction

\footnotetext{
${ }^{6} \mathrm{http}: / /$ twitter.com

${ }^{7}$ http://jilltxt.net/?p=184

${ }^{8} \mathrm{Par}$ exemple http://news.cell.com/cellreports/ pour l'éditeur de Cell, http://blogs.nature.com/ pour l'éditeur de Nature et http://thenode.biologists.com/ pour l'éditeur de Development.

${ }^{9}$ Par exemple http://loop.frontiersin.org/ pour l'éditeur Frontiers et http://blogs.plos.org/ pour l'éditeur PLoS.

${ }^{10} \mathrm{http} / / /$ listserver.ebi.ac.uk/mailman/private/pombelist/2009-October/000613.html
} 
de leurs mérites, faire la démonstration de son expertise sur un blog peut aider à être reconnu comme digne d'intérêt. Si un blogueur se prend à susciter les commentaires de ses lecteurs et interagir en ligne avec eux, il peut facilement glisser vers le réseautage. Ainsi, les blogueurs usent de plus en plus de ces canaux parallèles (aux interactions sociales classiques) pour entrer en contact avec des scientifiques qui leur ressemblent ou complémentaires, jusqu'à mener éventuellement des projets de recherche collaboratifs qui auraient semblé impossibles auparavant [Patil, 2009a]. Dans le pur esprit du web 2.0, ces collaborations sont éphémères et le plus souvent s'arrêtent une fois le projet amené à son terme. Certains chercheurs ont également noté que les blogs, qu'ils soient ouverts et réservés à un public restreint, peuvent servir d'outil de collaboration au sein d'une équipe de recherche, lui permettant de suivre l'avancée d'un projet et de construire une mémoire collective comprenant non seulement les idées originales (sous forme de billets de blog) mais aussi les commentaires et discussions qu'elles ont initié [Bradley, 2007].

Les blogueurs qui rédigent leurs «notes de terrain en public » [Wakeford, 2008] tirent parti des commentaires de leurs lecteurs, qui apportent des points de vue nouveaux et peuvent améliorer le travail de recherche... bien avant que cette pratique soit formalisée par le « post-publication peer review » mis en œuvre par certaines revues. L'exemple du philosophe John S. Wilkins est révélateur : son blog ${ }^{11}$ de philosophie de la biologie attire un large public intéressé par des sujets allant du concept d'espèce à l'institution académique en passant par le mouvement créationniste. En 2006, il publia une série de cinq billets de blog portant sur une recherche en cours consacrée aux concepts et causes de l'existence d'espèces microbiennes ${ }^{12}$. Ses billets ont reçu sept commentaires, un chiffre modeste mais significatif au commencement des blogs de science. Cependant, les commentaires renvoyaient vers des ressources additionnelles et alimentèrent une discussion embrassant un large spectre pluridisciplinaire allant de la philosophie à la microbiologie. Dans son article finalement publié en 2007 dans la revue Studies in History and Philosophy of the Life Sciences, Wilkins crédita ses «lecteurs de blog pour leurs commentaire et discussion».

J'ai pu bénéficier moi-même de la diversité des disciplines représentées au sein du lectorat moyen d'un blog de science. Par exemple, alors que je bloguais mes réflexions sur les manières de représenter la « science en train de se faire » plutôt que la « science faite », j'encourageai mes lecteurs à commenter et discuter abondamment ce billet ${ }^{13}$. Certains commentèrent appelèrent des clarifications, tandis que d'autres suggérèrent des exemples et des études que je ne connaissais pas. Un commentateur nota avec justesse que ma réflexion était écrite du point de vue d'un sociologue des sciences et il me renvoya vers des études du champ de la muséologie. J'élargis ainsi ma recherche et réussis à généraliser et renforcer mon argumentation.

On appelle «crowdsourcing » le fait de s'en remettre aux lecteurs ou simplement à ses contacts en ligne (plus il y en a, mieux c'est) pour répondre à une question, recommander des ressources pertinentes ou apporter un bout de leur expertise. Que la question soit directe (« Est-ce que cet article scientifique tout juste publié vaut quelque chose ?») ou plus complexe («Que dois-je lire de pertinent pour m'aider dans ce que je fais ? »), la combinaison de tant d'avis fournit une « information filtrée par le collectif » qui est moins sujette aux biais individuels. Ce peut être extrêmement utile dans un monde où un individu ne peut espérer se renseigner à la source sur tous les sujets qui l'intéressent.

Des sites web comme Research Blogging ${ }^{14}$ ou Altmetric ${ }^{15}$ puisent dans ce savoir collectif pour faire ressortir les actualités scientifiques les plus discutées dans la blogosphère. Patil et Siegel [2009b]

\footnotetext{
${ }_{11}^{11}$ http://evolvingthoughts.net

$12 \mathrm{http}: / /$ scienceblogs.com/evolvingthoughts/2006/06/on_microbial_species.php

${ }_{13}^{13} \mathrm{http}: / / \mathrm{www}$. enroweb.com/blogsciences/index.php?2008/05/31/261

${ }_{15}^{14} \mathrm{http}: / /$ researchblogging.com

${ }^{15} \mathrm{https}: / / \mathrm{www}$.altmetric.com
} 
suggèrent qu'ils sont souvent plus pertinents que la manière traditionnelle de filtrer la littérature publiée en se basant sur les recommandations des experts (typiquement les «choix de la rédaction » proposés par les grandes revues scientifiques), les habitudes de lecture et les moteurs de recherche.

\section{Crowdsourcing et sciences participatives}

Au sens strict, le crowdsourcing est un principe qui s'applique à des problèmes pour lesquels l'expertise est rare et la probabilité de réussite individuelle est faible, ou la somme des contributions donne un résultat meilleur qu'une contribution isolée fut-ce celle d'un expert. Pour cela, il faut pouvoir découper le travail à accomplir en un très grand nombre de sous tâches simples et répétitives (une cathédrale ne pourra jamais être construite par crowdsourcing ; mais une encyclopédie comme Wikipédia l'est).

À ce titre, la méthode scientifique qui procède par collecte de données se prête au crowdsourcing, à l'instar des astronomes amateurs qui écument le ciel depuis la Révolution scientifique à la recherche de corps célestes non identifiés. Plus proche de nous, il faut citer le premier recensement d'oiseaux des jardins organisé par la Société ornithologique Audubon aux États-Unis à Noël 1900 (et répété chaque année depuis), jusqu'au lancement du jeu de découverte scientifique FoldIt en 2008 pour faire replier des modèles 3D de protéines comme dans un puzzle, en passant par les célèbres initiatives de calcul scientifique distribué SETI@Home, Rosetta@Home et Stardust@Home de 1999 à 2006.

Le projet européen Citizen Cyberlab porté par un consortium a permis de distinguer trois formes de contributions de la « foule » (traduction littérale de crowd) dans le contexte des sciences participative : la détection (recueil de mesures physiques, photographies de phénomènes ou d'espèces naturels...), la cognition (saisie de texte à numériser, annotation d'images...) et le calcul (donner du temps de processeur de son ordinateur personnel, comme pour les projets @Home mentionnés précédemment).

\section{Bloguer pour la médiation scientifique}

Lors d'une table ronde sur les blogs de science, la blogueuse américaine sous pseudonyme Grrl Scientist témoigna ainsi des origines variées de son lectorat ${ }^{16}:$ «deux tiers de mes visites proviennent de serveurs en .edu [sites universitaires américains] ou .gov [sites gouvernementaux américains]. Principalement depuis des établissements d'enseignement. Des personnes du Sénat lisent mon blog, de nombreux éditeurs lisent mon blog, quelques avocats... des personnes qui viennent de Wall Street. J'ai des lecteurs âgés de 15 ans mais aussi des grands-parents. Il y a même des classes qui lisent mon blog régulièrement. » Un autre blogueur, Brian Switek, répond qu'il classe ses lecteurs selon le type de commentaires qu'il reçoit : des personnes qui étudient les sciences, celles qui veulent mieux comprendre les sciences, et celles qui cherchent une explication toute prête... réparties à parts égales.

La diversité du public est indéniablement un argument fort en faveur de l'utilité des blogs de science. Là où les musées et festivals de science touchent un public généralement bien connu, n'importe quel internaute peut se retrouver à lire un blog de science suite à une recherche Google ou en flânant sur le web, sans parler des lecteurs fidèles qui ont pris leurs habitudes dans la blogosphère scientifique. Les blogs se prêtent donc naturellement à la vulgarisation des sciences pour un public large. Cette mise en public peut prendre autant de formes que les blogs eux-mêmes, allant du commentaire de l'actualité scientifique rapportée par les grands médias au témoignage de la recherche en train de se faire dans le laboratoire du blogueur. D'autres excellent dans les articles

\footnotetext{
${ }^{16} \mathrm{http} / / / \mathrm{www}$. underthemicroscope.com/index.php?option=com_content\&task=view\&id=87\&Itemid=54
} 
didactiques (par exemple pour expliquer les dinosaures à plumes ${ }^{17}$, la découverte du boson de Higgs $^{18}$ ou la détection des ondes gravitationnelles ${ }^{19}$ ) ou prennent le prétexte d'un film récent ${ }^{20}$ ou d'une décision de justice ${ }^{21}$ pour mettre en évidence la science sous-jacente. L'histoire des sciences est souvent de la partie, avec un ton qui peut être neutre, enjoué, exaspéré, amical... [Goldstein, 2009].

Les blogs de science peuvent également s'approcher de ce qu'on attendrait des journalistes scientifiques. D'une part, parce que certains blogueurs sont en réalité journalistes professionnels. D'autre part, parce que les journalistes ont de moins en moins d'informations exclusives arguait le blogueur de longue date Bora Zivkovic en mars $2008^{22}$ : les annonces de dernière minute comme la mort d'un chercheur célèbre ne demandent que peu de détails et peuvent très vite être reprises par des internautes non journalistes; les nouvelles relatives à une publication scientifique ou un colloque sont diffusées largement par les chercheurs qui ont accès aux mêmes sources d'information ; l'analyse d'une actualité est une seconde nature pour les blogueurs qui connaissent le domaine et savent faire le tri dans les informations qu'ils lisent et entendent; et même les reportages d'investigation ne sont plus l'apanage des journalistes. Ainsi, des blogueurs ont mis au jour un cas de plagiat en paléontologie des dinosaures ${ }^{23}$ et ont joué un rôle important dans la libération des infirmières bulgares condamnées à mort en Libye pour avoir prétendument infecté des centaines d'enfants avec le virus du SIDA [Butler, 2006].

Malgré tout, il existe deux grandes différences entre les blogs de science et les médias scientifiques traditionnels. La première différence est que les blogs sont le point de départ de conversations, qui se développent d'un blog à un réseau social avec la participation active des lecteurs commentateurs. Il faut reconnaître que c'est aussi de plus en plus le cas des médias traditionnels, mais les blogs ont montré la voie. Sur les blogs de science, les lecteurs s'engagement activement, posent des questions, expriment leur désaccord, ne laissant pas le blogueur pontifier dans son coin mais le confrontent à des problématiques du terrain. La seconde différence est que les blogs sont ouverts à quiconque est intéressé par le sujet. Ils donnent la même voix aux naturalistes amateurs, patients curieux de leur maladie, curieux de science et quidam. Plutôt qu'une communication de haut en bas qui instaure de fait une barrière entre l'expert et le profane, les blogs composent un mélange de voix et de points de vue sur des sujets qui sont souvent plus complexes et moins univoques que les scientifiques l'imaginent — ce que les contributeurs de Wikipédia vérifient également chaque jour.

Dès ses débuts, le web a très vite développé une tradition de forums de discussion, où les personnes partageant un intérêt commun engagent la conversation. Cependant, et malgré leur utilité avérée, ces forums tendent à recréer les frontières traditionnelles entre les communautés et les niveaux d'expertise, et présentent une barrière à l'entrée non négligeable. En comparaison, il nous semble que les blogs trouvent dans la force de leurs récits et leur style informel, moins intimidant, les ingrédients d'une discussion plus ouverte à laquelle le lecteur passif ou de passage pourra facilement prendre part.

\section{Bloguer sans frontières}

\footnotetext{
${ }^{17}$ Partie 1 : http://ssaft.com/Blog/dotclear/?post/2009/05/11/Les-dinosaures-agrave\%3B-plumes-\%3A-Lemodegrave\%3Ble ; Partie 2 : http://ssaft.com/Blog/dotclear/?post/2009/05/19/Les-dinosaures-agrave\%3B-plumes\%3Ace-qu-en-disent-les-moleacute\%3Bcules ; Partie 3 : http://ssaft.com/Blog/dotclear/?post/2009/05/27/Les-dinosauresagrave\%3B-plumes\%3A-les-fossiles

${ }_{18} \mathrm{http} / / /$ lasciencepourtous.cafe-sciences.org/articles/le-boson-de-higgs-decouvert-au-cern/

${ }^{19} \mathrm{http}: / /$ kidiscience.cafe-sciences.org/articles/nous-voyons-enfin-la-gravite/

${ }^{20}$ Par exemple http://www.sciencesaucinema.fr/

${ }^{21}$ Par exemple http://buchanan.blogs.nytimes.com/2007/05/16/the-prosecutors-fallacy

$22 \mathrm{http} / / /$ scienceblogs.com/clock/2009/03/defining_the_journalism_vs_blo.php

${ }^{23} \mathrm{http}: / /$ scienceblogs.com/tetrapodzoology/2008/01/aetosaurs_and_whistle-blowing.php
} 
Pour la clarté de la démonstration, j'ai distingué deux objectifs principaux des blogs de science, quand bien même les blogs se caractérisent par le brouillage des catégories. Ainsi, le genre du blog de science combine souvent un point de vue sur le contexte autant que sur le contenu d'une recherche scientifique, brouillant la distinction entre la science comme processus et la science comme corpus de résultats. Le témoignage de la chercheuse Jill Walker sur ses premières années de blogueuse permet d'illustrer ce principe [Walker, 2006] : «Ce que je finis par écrire fut un genre hybride, mon style préféré de blog de recherche : le blog qui discute à la fois du contenu de la recherche, des idées elles-mêmes, et qui discute également du processus et de la pratique de la recherche ». Cette approche réflexive est encore rare dans les autres canaux de diffusion des sciences.

Scott L. Montgomery a noté que «non seulement le numérique ouvre la science à une participation plus large et de nouvelles formes d'expression, mais ce faisant il montre que les frontières dont on considérait qu'elles distinguaient l'essence de l'aventure scientifique des autres domaines comme la politique, l'économie ou la culture contemporaine au sens large, sont largement des illusions » [Montgomery, 2009]. Au lieu de faire un choix artificiel entre un public restreint de pairs auquel communiquer le processus de production des connaissances, ou disséminer la science à des audiences plus neuves, les blogueurs peuvent s'exprimer selon différentes règles sur différents sites. Ils construisent ainsi un public mixte sans a priori sur les personnes qui seront intéressées, les personnes qui liront et les personnes qui réagiront. Ils abattent les nombreux murs de la tour d'ivoire académique et rendent « le flux d'idées qui circulent dans une institution ou un champ disciplinaire encore plus accessible au public ${ }^{24} \gg$, afin que chacun en prenne connaissance, en tire profit ou le discute. En science comme dans n'importe quel champ académique, cette rupture peut être majeure. Ainsi, le professeur Douglas A. Berman estime que seulement une demi-douzaine de ses articles environ, sur plus de 50 articles et commentaires de droit, ont été cité dans des décisions de justice, alors que son blog a été cité dans plus d'une douzaine d'affaires judiciaires, « dont une opinion dissidente lors d'une décision historique de 2005 de la Court suprême des Etats-Unis ${ }^{25}{ }$.

La science en train de se faire offre souvent une perspective inattendue sur la pratique et la théorie scientifique, en révélant leur contingence et leur chemin sinueux, nous rappelant ainsi que par définition la science ne peut jamais être parfaite ou absolue. Les blogs de science nous permettent de jeter un œil par la porte entrouverte du laboratoire et de réaliser que la marche des sciences est plus importante pour nos vies que la « science froide » consacrée par l'institution. À l'heure où le changement climatique est mis en doute par des groupes d'intérêt, où les technologies d'édition du génome posent des questions éthiques et où la communauté scientifique s'interroge sur les fraudes, la qualité des données et la reproductibilité de ses résultats, la science ne peut plus attendre que le savoir soit stabilisé avant de le partager. Dans la société du risque traversée par des enjeux complexes, il y a peu de chance que la science soit sanctuarisée alors même que les chercheurs s'engagent dans des débats houleux et de négociations sans fin. Les blogs, eux, n'entretiennent pas cette illusion.

La personnalisation extrême des blogs de science, qui sont à l'image de leur auteur, et leur subjectivité assumée aident à faire passer le contenu factuel au second plan du méta-discours qui l'accompagne : qui dit quoi, dans quel contexte, selon quelles hypothèses... Ceci a longtemps été un point aveugle de la communication scientifique, en dépit des études sociologiques qui ont montré à quel point «l'information des coulisses » (backstage information) est indispensable pour appréhender un débat scientifique [Martin, 2000]. De la même façon, les pages d'historique et de discussion de l'encyclopédie en ligne Wikipédia rendent visible l'échafaudage de cet édifice

\footnotetext{
${ }^{24} \mathrm{http} / / / \mathrm{www} . l u k e g i l m a n . c o m / b l a w g / 2006 / 07 / 31 /$ blogging-in-higher-education-the-invisible-college ${ }^{25} / \mathrm{dem}$
} 
intellectuel.

Notons que le dialogue, forme consubstantielle aux blogs de science, est courant également dans la recherche et la médiation des sciences. Les pairs dialoguent en permanence entre eux pour construire leur intersubjectivité, et les initiatives des années 2000 visant à faire participer les citoyens dans les débats socio-techniques ont souvent pris la forme de débats publics. À ce titre, il me semble que les blogs de science s'imposent comme le canal idéal pour amener les scientifiques et chercheurs au plus près de leur public, tout en induisant une participation accrue de celui-ci. Plusieurs décennies après que la sociologie des sciences nous a convaincus que la communication scientifique formelle et la vulgarisation représentent les deux bouts d'un continuum plutôt qu'une dichotomie radicale [Shinn, 1985], il est plus que temps d'agir et d'explorer de nouvelles voies qui ne s'encombrent pas des catégories auxquelles nous sommes habitués.

\section{Quelques réussites}

Faut-il absolument donner des exemples de réussites ou « success stories » pour montrer l'intérêt des blogs de science ? C'est une demande qu'on ne peut ignorer, en dépit de ce paradoxe : le blog se construit dans une relation de long terme avec ses lecteurs, en développant une compréhension partagée de ce que veut dire faire de la recherche ou se soucier de la science au $21^{\mathrm{e}}$ siècle. Le blog ce sont des petites choses, presque intimes, à l'exception des quelques cas qui attirent l'attention. Pour reprendre les termes de Jill Walker [Walker, 2006] : «Les blogs sont intrinsèquement sociaux. Que vous ayez cinq lecteurs ou cinq cents importe peu, c'est le fait de savoir qu'on sera lu qui compte. »

Lorsque j'ai commencé à bloguer comme étudiant en master de sociologie des sciences en 2006, un de mes objectifs était de montrer aux lecteurs ce qu'est cette discipline, quels sont ses enjeux, ce qu'il essaye d'accomplir. Sur plusieurs centaines de billets j'ai couvert des fraudes scientifiques et la question de la responsabilité du chercheur, l'évaluation par les pairs et les mécanismes de la publication scientifique, les relations entre expertise scientifique et démocratie, les politiques de la technologie, la robustesse des résultats scientifiques ${ }^{26} \ldots$ Rien d'extraordinaire, juste ma tentative toute personnelle d'apporter un éclairage sur ces questions et de lancer des conversations à une échelle même modeste. Lors des mobilisations qui ont marqué l'année 2008-2009 en France, où les universitaires ont manifesté contre des décisions politiques qu'ils jugeaient contraires à la qualité de leur recherche et leur enseignement, la communauté scientifique a réalisé qu'elle n'avait qu'un petit poids dans l'opinion publique. Leurs arguments ne portaient pas, principalement parce que les enjeux étaient compliqués et parce qu'ils étaient victimes du syndrome de la « tour d'ivoire ». Ce diagnostic provoqua des discussions politiques sur la façon dont les blogueurs de science, en changeant doucement la façon dont ils sont perçus et en faisant entrer de plus en plus de lecteurs profanes dans leur monde, pouvaient faire bouger les choses ${ }^{27}$.

Le succès d'un blog de science se mesure d'abord par la satisfaction du blogueur envers sa production et son utilisation de l'outil. Ensuite vient le fait que ses billets sont cités, référencés par d'autres, commentés et partagés sur les réseaux sociaux - une mesure d'impact qui peut être comparée avec les métriques bibliométriques classiques dans l'évaluation de la recherche [Laursen, 2012]. Il est aussi stimulant et gratifiant d'observer simplement le format blog se développer et assister à autant de discussions riches et intelligentes. Ainsi, le blogueur français Tom Roud (un pseudonyme) publia en mars 2009 ses réflexions sur l'invention de l'agriculture et l'évolution des sociétés modernes ${ }^{28}$. Durant un mois, de nombreux internautes incluant un anthropologue, un

\footnotetext{
${ }^{26}$ http://www.enroweb.com/blogsciences

${ }^{27}$ See http://www.enroweb.com/blogsciences/index.php?2009/06/02/398

${ }^{28} \mathrm{http}: / /$ tomroud.com/2009/03/26/agriculture-economie-et-evolution
} 
chimiste, un chercheur en informatique et le responsable de la rubrique archéologique d'un magazine de vulgarisation... ont participé à la discussion et échangé des idées sur une question ouverte, posée pour le plaisir du débat. Avec cette saine motivation, tout ce qui provoque l'étonnement peut devenir une question gratuite qui, à partir d'un billet de blog, viendra bousculer l'état des connaissances ou contredire un article publié dans la littérature scientifique [Hadlington, 2009].

Je ne donnerai qu'un exemple de réussite liée à l'utilisation du blog pour la recherche. En janvier 2009, le mathématicien Tim Gowers a lancé un projet de collaboration à grande échelle en mathématique, appelé Polymath. Son principe : pour s'attaquer à un problème mathématique, Polymath engage un grand nombre de contributeurs, investissant chacun un temps non négligeable à la résolution du problème de manière à atteindre plus vite et plus intelligemment une solution, grâce à la diversité des créativités, compétences et connaissances des contributeurs. Après avoir identifié un problème qui pouvait se prêter à cette organisation, Gowers dut bâtir une communauté (en commençant par les lecteurs de son blog) et faire en sorte que ça fonctionne de manière collaborative, transparente et en ligne ${ }^{29}$. Le problème fut résolu en six semaines et l'expérience tellement bonne que la communauté mathématique mondiale lançait l'onzième problème Polymath à la date de rédaction de cet article ${ }^{30}$. Il faut noter que Polymath fait usage à la fois d'un blog et d'un wiki, c'est-à-dire un site que chacun peut éditer facilement comme Wikipédia. Là où le wiki permet d'organiser l'information et les nouvelles pistes de résolution du problème, le blog conserve le fil des essais-erreurs et de leurs auteurs. Comme le remarquait un participant se définissant comme «amateur avec seulement un diplôme de licence en mathématique ${ }^{31} »:$ : le format linéaire du blog était fantastique en ce qu'il permettait à mon inspiration de venir par l'observation du processus de recherche. Et le wiki était aussi génial puisque ces deux dernières semaines il m'a permis de rattraper tous les pré-requis mathématiques pour comprendre réellement ce qu'il se passait. [Une avancée importante du projet sous la forme d'une preuve mathématique fut] un grand moment pour moi car il cristallisa toute ma compréhension du projet — peu après j'y retournai et relus tous les commentaires pour mieux interpréter la discussion. Donc pour moi, la vraie valeur du projet était dans le fait d'écrire [sur un blog] les étapes intermédiaires, et pas simplement une fois qu'on a fini de travailler et qu'on a un article à publier ».

\section{Tous les scientifiques blogueront-ils?}

Après avoir présenté les nombreux avantages des blogs de science, on peut se demander si ce mode de communication se révèlera être l'équivalent du courrier électronique pour les laboratoires de recherche au $21^{\mathrm{e}}$ siècle. Dit autrement, est-ce qu'on verra de plus en plus de scientifiques remercier « les lecteurs du blog pour leurs commentaires et discussions » à la fin de leur articles académiques ? Et, prédiction mise à part, est-ce que c'est souhaitable ?

Le biologiste devenu vulgarisateur Jean-Marc Galan a offert un bon résumé de la situation sur son $\operatorname{blog}^{32}$. Pour lui, les scientifiques se divisent en deux catégories (même s'il y a en réalité un continuum entre les deux) : ceux qui pensent qu'on laisse plus de choses dehors que dedans en fermant la porte, et ceux qui préfèrent travailler dans leur coin par peur d'être plagiés ou doublés. En raison de cette différence fondamentale, les premiers seront plus enclins à bloguer (et à pratiquer une science 2.0) que les seconds. Cependant, dès qu'ils se mettant à bloguer, leur organisme de recherche entre en ligne de compte. Ils sont de plus en plus nombreux à encourager les blogs de

\footnotetext{
${ }^{29} \mathrm{http}: / /$ scienceblogs.com/christinaslisrant/2009/08/an_overview_of_the_polymath_pr.php

${ }_{31}^{30} \mathrm{http}: / /$ polymathprojects.org

${ }^{31} \mathrm{http} / / /$ gowers.wordpress.com/2009/03/10/polymath1-and-open-collaborative-mathematics/\#comment-2710

32 http://www.recherche-en-cours.org/REC/Blogcast/Entrées/2008/9/12_Science_2.0_-_2_3.html
} 
science voire à offrir des outils de blogs à leurs chercheurs, à l'instar de l'Oxford Internet Institute ${ }^{33}$ et de l'École polytechnique fédérale de Lausanne ${ }^{34}$ [Batts, 2008]. Avec de telles validations par la hiérarchie, même les chercheurs récalcitrants pourraient rejoindre la conversation. Roger Pielke, Jr., du blog Prometheus ${ }^{35}$ affilié au Center for Science and Technology Policy Research de l'université du Colorado, estime par exemple qu' « enraciner le blog dans l'institution académique augmente sa légitimité et sa diffusion » [Batts, 2008].

Cependant, cette tendance soulève plusieurs questions. Premièrement, une fois institutionnalisés, les blogs doivent subir un contrôle qualité et leur succès est quantifié [Batts, 2008] — avec le risque que ces indicateurs soient un nouvel avatar des métriques bibliométriques, et que les métriques nouvelle génération basées sur l'audience, la diffusion... souffrent finalement des mêmes biais que les très critiqués facteur d'impact et nombre de citations. Deuxièmement, le blog est par essence une pratique ascendante et un témoignage continu de l'autonomie du corps académique, composé d'hommes et de femmes plutôt que d'institutions et de gouvernement. Comme le notait le blogueur et historien des sciences Thomas Söderqvist, en reprenant à son compte des catégories issues des sciences politiques : les blogs de science écrits avec un point de vue singulier et rédigés à la première personne représentent la multitude, tandis que la communication scientifique institutionnelle incarnant un point de vue dominant représente l'Empire ${ }^{36}$. Ainsi, l'influence des dynamiques de pouvoir et des conflits au sein du monde scientifique sont au cœur des débats sur le futur des blogs de science et ne devraient pas être écartés facilement ${ }^{37}$. En fait, plutôt qu'aux incitations institutionnelles, les chercheurs pourraient être plus sensibles aux incitations communautaires. La courte histoire des blogs de science [Blanchard, 2016] a montré que les communautés comme ScienceBlogs ${ }^{38}$ en anglais et le Café des sciences ${ }^{39}$ en français ont bâti leur succès en offrant un mélange varié de blogs de science, augmentant ainsi la visibilité de l'ensemble, maintenant la dynamique et permettant de trouver des synergies entre les compétences et les intérêts individuels. Dans le cas du Café des sciences, cela a permis à des membres de bloguer en dehors de leur zone de confort et oser attaquer de nouveaux sujets - en sachant qu'un collègue pourrait ajouter leur grain de sel dans la discussion ou corriger les erreurs - et a facilité l'écriture de billets à plusieurs mains. Le succès évident des communautés de blogs de science construites « par le bas » est un facteur important dans les nouvelles vocations de blogueurs.

Un moyen terme entre la marginalisation et l'institutionalisation des blogs de science se trouve chez Shalley A. Batts et al· [Batts, 2008] : «si des groupes de blogueurs devaient développeur leurs propres initiatives et chercher ensuite une reconnaissance institutionnelle, ils pourraient être capables de discuter de science comme ils l'entendent tout en prouvant à l'institution - comme ils s'échinent déjà à le prouver à leurs lecteurs et leurs pairs - que les conversations dans lesquelles ils s'engagent en valent la peine. (...) En ouvrant un dialogue franc et ouvert sur leurs objectifs communs, les blogueurs et leurs institutions peuvent faire progresser ensemble la qualité et le champ des conversations mondiales sur la science auxquelles nous participons tous et dont nous dépendons ». Une autre voie vers la normalisation repose dans l'expression « carnet de recherche en ligne », qui éloigne les blogs de science de l'image du carnet intime adolescent ou de médium polémique qui leur est associée. C'est le choix sémantique de la plateforme de blogs en SHS Hypothèses $^{40}$, placée sous la responsabilité conjointe de deux universités, du CNRS et de l'EHESS, qui donne à l'outil une autre image et le rend compatible avec l'institution sans le dénaturer.

\footnotetext{
${ }^{33}$ http://people.oii.ox.ac.uk

${ }^{34} \mathrm{http}: / / \mathrm{blog}$ s.epfl.ch

${ }^{35} \mathrm{http}: / / \mathrm{sciencepolicy}$. colorado.edu/prometheus

${ }^{36} \mathrm{http}: / / \mathrm{www}$. corporeality.net/museion/2008/07/31/science-blogging-science-communication-and-the-multitude

${ }^{37} \mathrm{http}: / / \mathrm{www} . c 0 r p o r e a l i t y . n e t / m u s e i o n / 2008 / 09 / 24 /$ science-blogging-vs-institutionally-based-science-communication

${ }^{38} \mathrm{http}: / /$ scienceblogs.com

${ }^{39} \mathrm{http}: / / \mathrm{www}$. cafe-sciences.org

40 http://hypotheses.org
} 
Dans ces conditions, que faut-il penser des chercheurs et scientifiques qui ne succomberont pas aux blogs de science dans le futur? Observera-t-on une différence entre ceux qui en on et ceux qui n'en ont $_{\text {pas }}{ }^{41}$ ? Probablement pas : ceux qui ne sautent pas le pas ne verront pas leur qualité scientifique diminuer, et il y aura toujours des bons chercheurs qui ne bloguent pas. Cependant, la différence pourrait se jouer dans la société, qui aurait à perdre des externalités positives des blogs de science si leur pratique ne se généralisait pas. En économie, la notion d'externalité positive dénote l'impact d'une transaction sur un tiers qui n'est pas directement impliqué. Par exemple, le traitement ignifuge d'une maison améliore également la sécurité incendie des maisons voisines. J'émets l'hypothèse qu'une partie de, sinon toute, la valeur des blogs de science repose dans leurs externalités : ils ne bénéficient pas seulement au chercheur qui s'exprime [Wakeford, 2008] mais aussi aux autres scientifiques et au grand public. Des valeurs qui préoccupent la société dans son ensemble comme l'ouverture, l'accès et l'engagement sont bien représentées par les blogs de science... et à mon sens notre société serait plus vivable si tous les chercheurs bloguaient.

\section{Conclusion}

Même si les blogs de science ne deviennent pas aussi omniprésents que le courrier électronique, ils ne vont pas disparaître : je crois qu'ils vont devenir en une décennie une pratique courante et acceptée. Ce qui aura un prix : symboles de la liberté d'expression, les blogs de science ont aussi leurs controverses, opinions à l'emporte-pièce et leurs billets paresseux. Ils sont d'efficaces machines à rumeurs, comme le montre l'histoire de Tomasso Dorigo [Chalmers, 2009]. Physicien des particules et blogueur, Dorigo avait commenté en janvier 2007 un petit pic observé dans les données du Fermilab américaine, dont il suggéra sur son blog qu'il pouvait être un signe du très recherché boson de Higgs ${ }^{42}$. Le magazine de vulgarisation New Scientist tira de cette nouvelle un article de deux pages intitulé « Aperçu de la particule de Dieu », puis ce fut le tour de publications à grand tirage comme The Economist et Wired. Alors que la nouvelle se répandait dans la blogosphère, le résultat relaté par Dorigo n'était même pas encore passé au crible des 500 collaborateurs de cette expérience à laquelle il participait. Il essaya tant bien que mal de prendre ses distances avec cette interprétation en arguant sur son blog que la faible significativité statistique de ce pic était mal représentée par le journaliste du New Scientist ${ }^{43}$, mais fut critiqué fortement par ses collaborateurs qui tenaient à leur processus strict de vérification et divulgation des résultats.

La blogosphère est également peuplée par une faune de lecteurs et commentateurs que les blogueurs ont appris à gérer ${ }^{44}:$ le «flamer » qui envoie des commentaires délibérément hostiles et des insultes ad hominem; le « troll » qui laisse des commentaires non pertinents, controversés ou hors-sujets ; le « kook » dont les messages se succèdent sans lien apparent avec la réalité ; le « shill » dont les messages dissimulent un groupe ou une organisation cachés, généralement en porte-à-faux avec le sujet de discussion.

En réalité, nous apprenons déjà à gérer ces intrus et à s'y adapter. Ils font partie de ce mouvement de fond qui nous force à repenser les pratiques de recherche, sans cesse changeantes. Que ce soit dans la manière dont les priorités scientifiques sont établies ou dont naissent les collaborations, la science vivra dans son siècle, comme elle l'a toujours fait. Un jour, nos enfants riront peut-être en apprenant que le blog fut considéré à ses débuts comme une pratique risquée, une perte de temps, ou au mieux un hobby - mais ce temps n'est pas encore arrivé. Les doctorants et jeunes chercheurs se font parfois rappeler à l'ordre par leurs directeurs ou encadrants : « arrête de bloguer et concentre-toi sur ton travail !». C'est aussi une des raisons pour lesquelles tant de blogueurs

\footnotetext{
${ }^{41} \mathrm{http}: / /$ wiser-u.net/blog/2009/06/17/would-ramanujan-have-a-blog

${ }^{42} \mathrm{http} / / /$ dorigo.wordpress.com/2007/01/19/a-21-sigma-eccess-of-mssm-higgs/

${ }^{43} \mathrm{http}: / /$ dorigo.wordpress.com/2007/03/01/the-mssm-higgs-signal-buried-in-my-plot

${ }^{44} \mathrm{http}: / / \mathrm{www}$. searchlores.org/trolls.htm
} 
écrivent sous pseudonyme, présentant les avantages de l'anonymat (leur véritable identité est cachée) sans certains de ses inconvénients (comme le fait de ne pouvoir être reconnu et bâtir une réputation à partir de ses actions en ligne) ${ }^{45}$. Voilà encore une pratique disruptive dans le monde scientifique classique, qui juge généralement des mérites d'un travail en s'attachant également à son auteur.

J'espère que ce chapitre a apporté un éclairage positif sur les blogs de science et motivera des chercheurs, universitaires, enseignants et citoyens du monde à explorer la blogosphère ( $c f$. les recommandations de lecture en annexe). Un moyen simple de se mettre à bloguer consiste à bloguer pour soi-même. En tant qu'outil personnel de gestion des connaissances, le blog nous force à organiser l'excès d'information que nous recevons et à partager son « flux de pensée » dans des billets horodatés. Quelques règles de base vous aideront à bien vous lancer ${ }^{46}$ : choisissez un hébergeur ou une plateforme de blogs réputé, protégez votre vie privée et celle de vos collègues et vos proches, essayez de publier deux billets par semaine et faites des liens vers vos sources. Alors immanquablement, un internaute tombera sur votre blog et viendra commenter pour abonder dans votre sens, donner des informations complémentaires ou apporter la contradiction. Vous vous retrouverez très vite engagés dans des conversations inespérées, sur les sujets qui comptent pour vous. Et il se peut que vous y preniez goût...

\section{Remerciements}

Ce texte est une traduction révisée et augmentée de «Science blogs in research and popularization of science: why, how and for whom? », In Moira Cockell et coll. (dir.), Common Knowledge: The Challenge of Transdisciplinarity, Lausanne : EPFL Press, 219-232 (2011), reproduit avec l'autorisation de l'éditeur. Je remercie Christina C. Pikas et Moira Cockell d'avoir rebondi sur mes idées, proposé de nouvelles pistes de réflexion et enrichi la première version de ce texte. Je suis redevable à François Taddéi et Livio Riboli-Sasco pour l'interprétation de la chaîne "Why blog ? ». Je suis reconnaissant à la communauté du Café des sciences pour son énergie, qui donne envie de repousser les limites du blog de science, et sa réflexivité. Je reste seul responsable des opinions exprimées dans ce chapitre et des erreurs qui pourraient subsister.

\section{Bibliographie}

Batts (Shelley A.), Anthis (Nicholas J.), Smith (Tara C.), « Advancing science through conversations: Bridging the gap between blogs and the academy », PLOS Biology, vol. 6, $n^{\circ}$, 2008, p. e240.

Blanchard (Antoine), «Quand la culture scientifique s'affranchit sur le web : l'exemple des blogs de science en français (2003-2014) », dans Poirrier (Philippe) (dir.), Histoire de la culture scientifique en France : institutions et acteurs, Dijon, Presses universitaires de Dijon, 2016.

Bradley (Jean-Claude), « Open notebook science using blogs and wikis », Nature Precedings, 2007 Butler (Declan), «Bloggers rally for liberation of the 'Tripoli Six' », Nature.com, 2006 Chalmers (Matthew), «Communicating physics in the information age », p. 67-80 dans Holliman (Richard), Thomas (Jeff), Smidt (Sam), Scanlon (Eileen), Whitelegg (Elizabeth) (dir.), Practising science communication in the information age: Theorising professional practices, Oxford, Oxford University Press, 2009.

Claessens (Michel), Science et communication pour le meilleur ou pour le pire ?, Paris, Quae, 2009.

Commission européenne, Special Eurobarometer 282 : Scientific research in the media, 2007.

\footnotetext{
${ }^{45}$ http://reassignedtime.blogspot.com/2008/04/pseudonymity-is-not-anonymity-duh.html

${ }^{46} \mathrm{http}: / /$ www.scidev.net/en/practical-guides/how-to-set-up-a-science-blog.html
} 
Feynman (Richard P.), Nobel Lecture: The Development of the Space-Time View of Quantum Electrodynamics, 1965.

Goldstein (Adam M.), «Blogging evolution», Evolution: Education and Outreach, vol. 2, $\mathrm{n}^{\circ}$ 3, 2009, p. 548-559.

Gregg (Melissa), «Feeling ordinary: Blogging as a conversational scholarship », Continuum.

Journal of Media \& Cultural Studies, vol. 20, $\mathrm{n}^{\circ}$ 2, 2006, p. 147-160.

Hadlington (Simon), « Peer review by live blogging », Chemistry World, 2009.

Laursen (Lucas), « Alternative Research Metrics », Sciencemag.org, 2012.

Martin (Brian), «Behind the scenes of scientific debating », Social Epistemology, vol. 14, $\mathrm{n}^{\circ}$ 2-3, 2000, p. 201-209.

Montgomery (Scott L.), «Science and the online world: Realities and issues for discussion », p. 8397 dans Holliman (Richard), Thomas (Jeff), Smidt (Sam), Scanlon (Eileen), Whitelegg (Elizabeth)

(dir.), Practising science communication in the information age: Theorising

professional practices, Oxford, Oxford University Press, 2009.

Patil (Chris), Siegel (Vivian), «Drinking from the firehose of scientific publishing », Disease

Models \& Mechanisms, vol. 2, n 3-4, 2009, p. 100-102.

Patil (Chris), Siegel (Vivian), « This revolution will be digitized: Online tools for radical

collaboration », Disease Models \& Mechanisms, vol. 2, n 5-6, 2009, p. 201-205.

Shinn (Terry), Whitley (Richard) (dir.), Expository science: Forms and functions of

popularisation, Dordrecht, Reidel Publishing Company, 1985.

Wakeford (Nina), Cohen (Kris), «Fieldnotes in public: Blogs for research », p. 307-326 dans

Fielding (Nigel), Lee (Raymond M.), Blank (Grant) (dir.), The SAGE handbook of online research methods, Londres, SAGE, 2008.

Walker (Jill), « Blogging from inside the ivory tower », p. 127-138 dans Bruns (Axel), Jacobs (Joanne) (dir.), Uses of blogs, New York, Peter Lang, 2006. 


\section{Annexe : Pour en savoir plus}

\section{Blogs de science distingués}

- The Globserver (lauréat des Golden Blog Awards 2014 dans la catégorie «Science/Recherche ») : http://theglobserver.com/

- Homo Fabulus (coup de cœur du jury de l'anthologie 2013 des blogues de science francophones) : http://homofabulus.com/

- Passeur de Sciences (lauréat des Golden Blog Awards 2015 toutes catégories) : http://passeurdesciences.blog.lemonde.fr/

- Podcast Science (lauréat des Golden Blog Awards 2012 dans la catégorie «Science/Recherche ») : http://www.podcastscience.fm/

- Science étonnante (coup de cœur du jury de l'anthologie 2014 des blogues de science francophones) : http://sciencetonnante.wordpress.com/

- La Science infuse (lauréat des Golden Blog Awards 2013 dans la catégorie «Science/Recherche ») : http://blog.science-infuse.fr/

Sans oublier la communauté du Café des sciences dont ils sont tous membres : http://www.cafesciences.org/

\section{Bibliographie}

L'auteur tient à jour une bibliographie des publications académiques portant sur les blogs de science : http://www.citeulike.org/user/Enro/tag/science-blog

Voici quelques références qui peuvent servir d'introduction à ce domaine de recherche :

- Colson (Vinciane), «Science blogs as competing channels for the dissemination of science news », Journalism, vol. 12, n 7, 2011, p. 889-902.

- Fausto (Sibele), Machado (Fabio A.), Bento (Luiz F.), Iamarino (Atila), Nahas (Tatiana R.), Munger (David S.), « Research Blogging: Indexing and Registering the Change in Science $2.0 »$, PLOS ONE, vol. 7, n 12, 2012, p. e50109.

- Kjellberg (Sara), «Blogs as interfaces between several worlds: A case study of the Swedish academic blogosphere », Human IT: Journal for Information Technology Studies as a Human Science, vol. 10, $\mathrm{n}^{\circ}$ 3, 2013.

- Le Deuff (Olivier), « Un blog scientifique », Médium, vol. 2013/3, n 36, 2013, p. 82-97.

- Mahrt (Merja), Puschmann (Cornelius), « Science blogging: an exploratory study of motives, styles, and audience reactions », Journal of Science Communication, vol. 13, $\mathrm{n}^{\circ} 3$, 2014, p. A05.

- Puschmann (Cornelius), Mahrt (Merja), « Scholarly Blogging: A New Form of Publishing or Science Journalism 2.0? », p. 171-181 dans Tokar (Alexander), Beurskens (Michael), Keuneke (Susanne), Mahrt (Merja), Peters (Isabella), Puschmann (Cornelius), van Treeck (Timo), Weller (Katrin) (dir.), Science and the Internet, Düsseldorf, Düsseldorf University Press, 2012.

- Ranger (Mathieu), Bultitude (Karen), «'The kind of mildly curious sort of science interested person like me': Science bloggers' practices relating to audience recruitment ». Public Understanding of Science, 2014.

- Riesch (Hauke), Mendel (Jonathan), « Science Blogging: Networks, Boundaries and Limitations », Science as Culture, vol. 23, n 1, 2013, p. 51-72. 
- Shema (Hadas), Bar-Ilan (Judit), Thelwall (Mike), « How is research blogged? A content analysis approach », Journal of the Association for Information Science and Technology, vol. 66, $\mathrm{n}^{\circ} 6,2015$, p. 1136-1149.

- Thorsen (Einar), «Blogging on the ice: Connecting audiences with climate-change sciences », International Journal of Media \& Cultural Politics, vol. 9, n 1, 2013, p. 87-101.

- Trench (Brian), « Scientists’ Blogs: Glimpses Behind the Scenes », p. 273-289 dans Rödder (Simone), Franzen (Martina), Weingart (Peter) (dir.), The Sciences' Media Connection Public Communication and its Repercussions, Dordrecht, Springer, 2012. 